\title{
Progresses on the intelligent construction and operation maintenance of urban under-lake tunnels
}

\author{
Jingfeng Wang ${ }^{1,2}$, Guang Liu ${ }^{1,2,3^{*}}$, Zhaodong Ding ${ }^{1,2}$, Hanbing Bian ${ }^{4}$, Huayan Yao ${ }^{1,2}$ \\ ${ }^{1}$ School of Civil Engineering, Hefei University of Technology, Hefei, China \\ ${ }^{2}$ Anhui Key Laboratory of Civil Engineering Structures and Materials, Hefei University of \\ Technology \\ ${ }^{3}$ Key Laboratory of Rock Mechanics in Hydraulic Structural Engineering (Ministry of Education), \\ Wuhan University, Wuhan, China \\ ${ }^{4}$ Laboratoire de Génie Civil et géo-Environnement, Université de Lille, 5900 Lille, France
}

\begin{abstract}
China is currently at an important stage of urbanization. In recent years, the number and scale of under-lake tunnels in southern cities of China are growing consistently, which brings opportunities and challenges in intelligent construction and operation maintenance. Due to the long length of the under-lake tunnel, the large volume of concrete to be cast, the complex environment of the buried section and the underground environment of the lake bottom, the hydration heat and stress crack have been the main challenges of the under-lake tunnel engineering in crack control and waterproofing aspect. Simultaneously, with the development of big data, intelligent construction, information technology, and other techniques, these techniques are gradually applied to the whole life cycle of tunnel engineering including survey, design, construction, operation, and maintenance. The construction of the lake tunnel project is setting off a new round of technological innovation. This paper systematically summarizes the technical breakthroughs and application of technologies such as intelligent construction and information technology in the construction of under-lake tunnels in China, and puts forward reasonable suggestions on the key technologies of intelligent construction and operation maintenance for urban under-lake tunnel engineering.
\end{abstract}

* Corresponding author: guang_liu@,hfut.edu.cn 


\section{Introduction}

The construction of under-lake tunnels has set off a new upsurge in the Chinese urbanization process. During the past decades, a number of under-lake tunnels are constructed in Chinese metropolises, such as Nanjing, Wuhan and Suzhou. There are many well-known under-lake tunnels in China, for instance, East lake, Tai lake, Xuanwu lake, and Dushu lake tunnels. The detailed information for some of the well-known under-lake tunnels in China is listed in Table 1.

Table 1. Some of the well-known under-lake tunnels in China

\begin{tabular}{|c|c|c|c|}
\hline Tunnel name & Overall length $(\mathbf{k m})$ & Width $(\mathbf{m})$ & Construction state \\
\hline East lake tunnel & 10.6 & $60-70$ & Completion \\
\hline Tai lake tunnel & 10.8 & 43.6 & Under construction \\
\hline Xuanwu lake tunnel & 2.7 & 32.0 & Completion \\
\hline Dushu lake tunnel & 3.5 & 30.2 & Completion \\
\hline
\end{tabular}

The under-lake tunnels have an important effect on the optimization of the urban road network system. The commuting time between two sides of the lake can be considerably reduced with the construction of under-lake tunnels. The commuting time from Liyuan to Guanggu which are two places on the two sides of the East lake shortens to 10 minutes from one hour after the East lake tunnel is built.

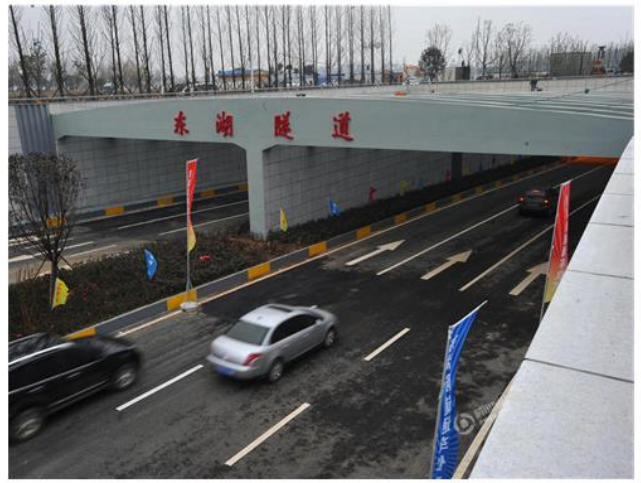

(a)

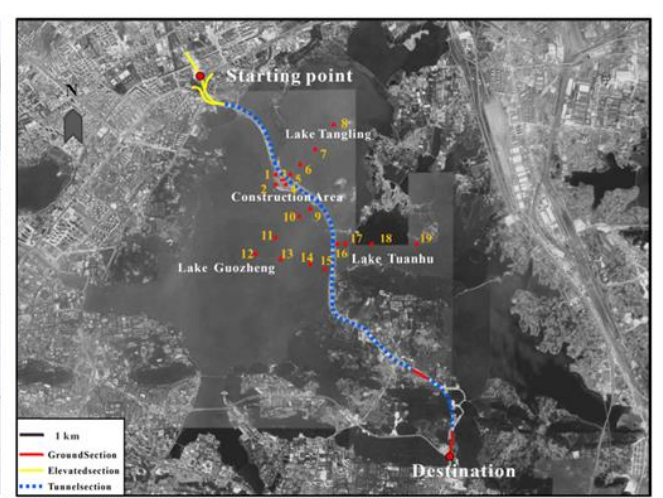

(b)

Figure 1. Figures of East lake tunnel in Wuhan. (a) The East lake tunnel opens to traffic. (b) A sketch map for the route of the East lake tunnel (Wang et al., 2016).

Due to the complexity and uncertainty of the under-lake tunnels, a large amount of data analysis and prediction are needed during the construction process. The intelligent algorithms, for example, artificial neural networks (ANNs), support vector machine (SVM) and Gaussian Process (GP), can be used to select the modeling parameters and predict the mechanical response of the under-lake tunnels (Darabi et al., 2012; Mahdevari et al., 2013; Li et al., 2017). A spreadsheet optimization technique coupled with the trained neural network model is used to study the reliability assessment of the settlement serviceability limit state by Goh and Hefney (2010). Recently, Liu et al. (2019) adopted a genetic algorithm and combined covariance Gaussian process regression coupled algorithm to execute displacement back analysis and predict displacement of surrounding rock in the initial monitoring period of tunnel construction. This prediction of surrounding rock 
displacement also can be extended to the long-term monitoring period after the training samples are collected.

This paper first reviews the main problems faced in the current construction of the underlake tunnels. Then the application of smart technology in the tunnel construction process is studied. A programmatic framework for the application of smart technology in the construction of the under-lake tunnels is established.

\section{Major challenges for urban under-lake tunnels}

There are many challenges in the whole life cycle of the construction and operation of urban under-lake tunnels. Due to limited space, this research mainly elaborates on leakage and concrete cracking.

\subsection{Water leakage in the under-lake tunnels}

A remarkable feature for the under-lake tunnels is the tunnels need to stretch under the lake, and the burial depth of the tunnels is highly dependent on the geological conditions of the bottom sediment in the lake. Therefore, for high permeability soils on the lake bottom, the tunnels may be subjected to great risk in water leakage.

As a matter of fact, the current status for the waterproofing of underground tunnels in China is not optimistic. This issue may be more severe for the under-lake tunnels because this underground structure has direct interaction with the lake. According to the statistics of China Building Waterproof Association (2013), the leakage rate of underground engineering in China is as high as $80 \%$. Compared with the collapsed construction accidents during the construction process, the underground leakage is generally slow and concealed. Therefore, the underground leakage attracts less attention of all parties until a devastating accident happened.

In 2013, China Building Waterproof Association and Beijing Zero Market Research and Analysis Company (2013) jointly released a sample survey of the National Building Leakage Survey Project Report, involving 1777 underground building samples from 28 cities. In the survey, 1022 underground structures had leakage problems in varying degrees. The leakage rate reached $57.51 \%$, in some cities such as Chongqing, Wuxi and other places, the leakage rate of underground buildings is as high as $100 \%$. According to statistics, the annual maintenance cost of water leakage in underground space in China accounts for more than $50 \%$ of the total maintenance cost. The problem of water seepage and water leakage in urban underground structures has become a common problem in construction. 


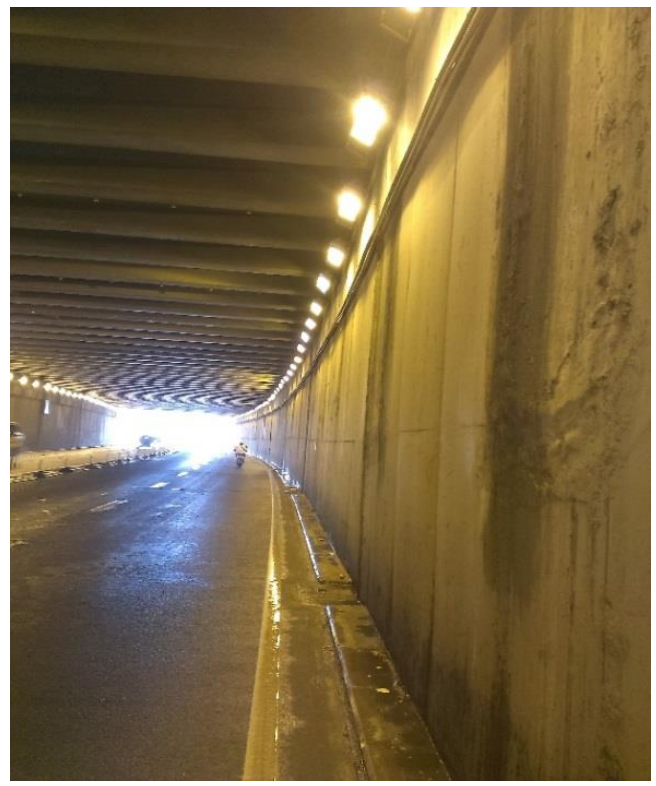

(a)

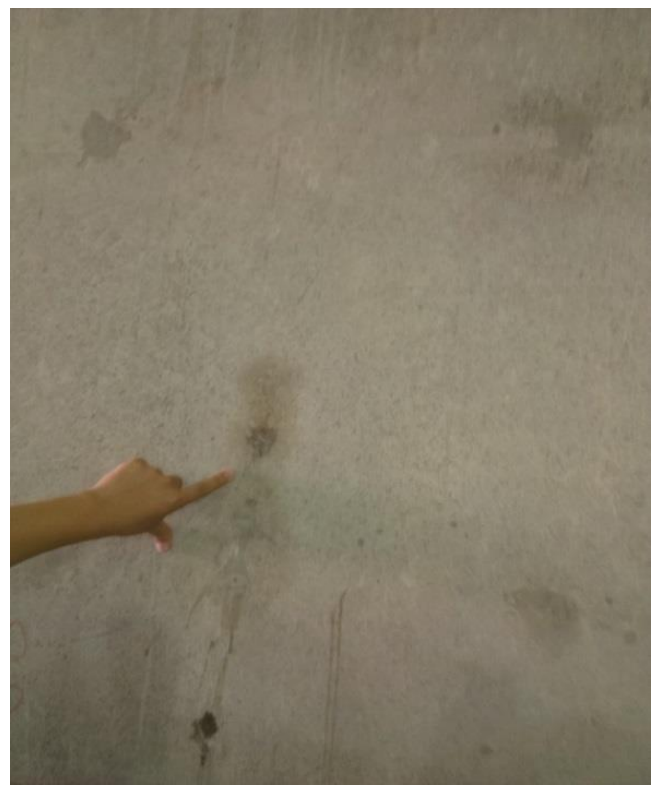

(b)

Figure 2. Leakage on the sidewall of an underground passage for the motor vehicles in Hefei, Anhui.

(a) An underground passage at Luyang district. (b) An underground passage at Shushan district.

\subsection{Cracking of the concrete}

The geological environment of the lake bottom tunnel project is significantly different from other construction projects. Due to the Huge pouring amount of concrete and complex underground environment at the bottom of the buried section, the crack propagation caused by temperature stress and uneven deformation can be found in underground structures. Cracking of tunnel concrete is a common problem in underground engineering (see Figure $3)$.

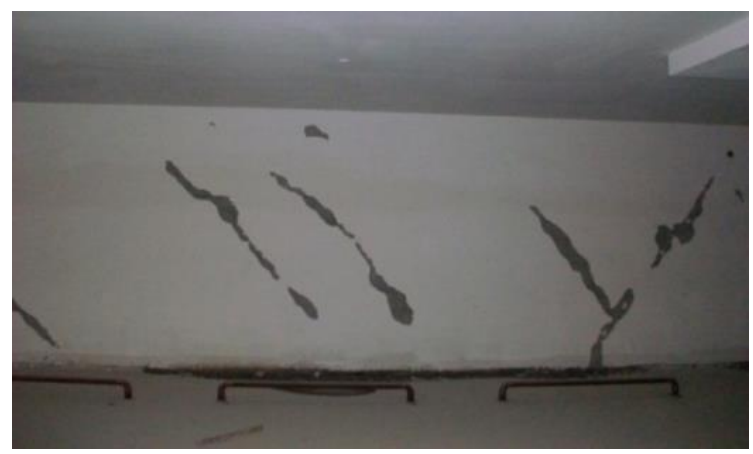

Figure 3. Cracks on a concrete shear wall 
There are two main reasons which cause the cracking of underground tunnels. One is that the uneven distribution of hydration heat of mass concrete, which causes temperature cracks. The other is due to the non-uniformity properties of the foundation soil, resulting in uneven settlement or deformation at different locations for the underground structure. It can be found from Figure 4 that the damage is concentrated on the sides of the sidewall once the left side has a vertical settlement of $10 \mathrm{~mm}$. This damage area is very likely to cause crack propagation in the concrete.

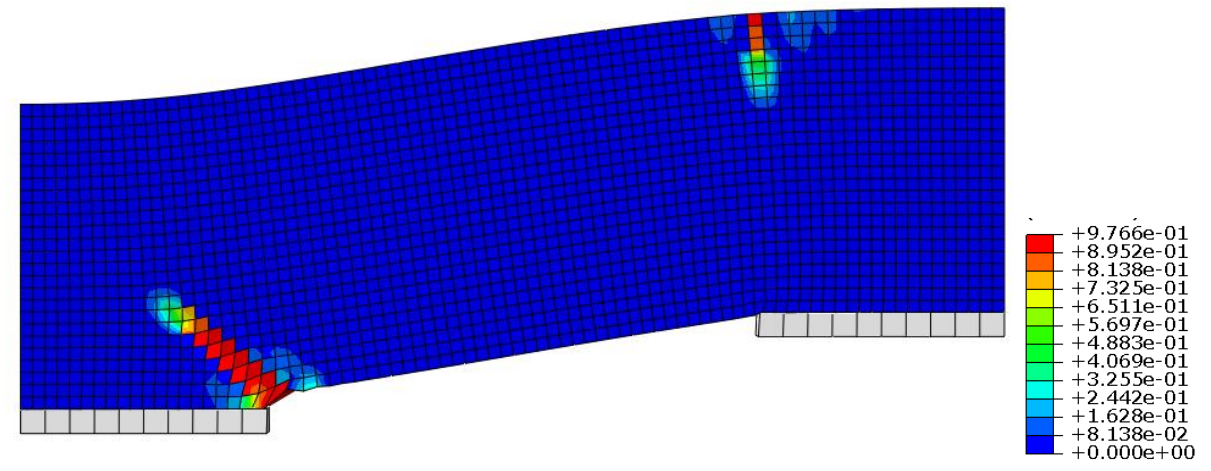

Figure 4. Damage distribution when the left the sidewall in an underground structure has a vertical settlement of $10 \mathrm{~mm}$. The damage degree is represented by a dimensionless damage factor shown the legend. The damage factor ranges from $0-1$. No damage is indicated by 0 and full damage indicated by 1 .

In addition, concrete cracking and leakages are two coupled physical processes (Davies et al., 2014; Liu et al., 2019). It is well known that uneven external forces (water pressure, earth pressure, frost heave force, insufficient bearing capacity, earthquakes, etc.) in the underground environment are applied to the structure, causing uneven deformation or even cracking of the structure, resulting in a large localized waterproof layer. This effect is more pronounced when the tunnel encounters an inhomogeneous deformation. Once the stress exceeds the allowable value, the internal microcracks initiate, propagate, and extend to form a series of fractures, which further destroys the waterproof structural system. Meanwhile, it is worth noting that the long-term leakage also accelerates the failure of concrete due to the strength degradation of concrete under water immersion conditions.

\section{Application of smart technology}

At present, technologies such as big data and artificial intelligence are developing rapidly. In the construction and operation of the under-lake tunnels, these smart technologies should be used for intelligent monitoring and information management.

As mentioned above, leakage and concrete cracking are two important problems in the construction of lake tunnels. These two problems exist in the entire life cycle including construction, construction, operation, and maintenance stage of the under-lake tunnel. These problems have a long time span, and they are difficult to detect. Therefore, we can make full use of the current smart technology for automated detection and information analysis, and implement intelligent monitoring of the life cycle for the under-lake tunnels.

As shown in Figure 5, the application framework can be divided into three parts: the data acquisition layer, the analysis layer, and the control layer. The data acquisition layer mainly 
collects the displacement, deformation, temperature, image and other data on the key positions in the stage of construction, operation and maintenance through high-precision cameras and high-sensitivity sensors. The analysis layer uses the collected displacement, stress and other data as learning samples through the neural network, machine learning, deep learning algorithm. After the sample training, the analysis layer can predict the displacement and stress in the next stage. The analysis layer can also process image data to identify locations of cracks and leakages, which further provides guidance for manual intervention. The control layer performs manual interventions to prevent leakages and crack propagation. The chemical grouting, spraying waterproof coatings are two common interventions for the leakage and cracking problems. In addition, the control layer will optimize the construction plan and building materials based on current monitoring data.

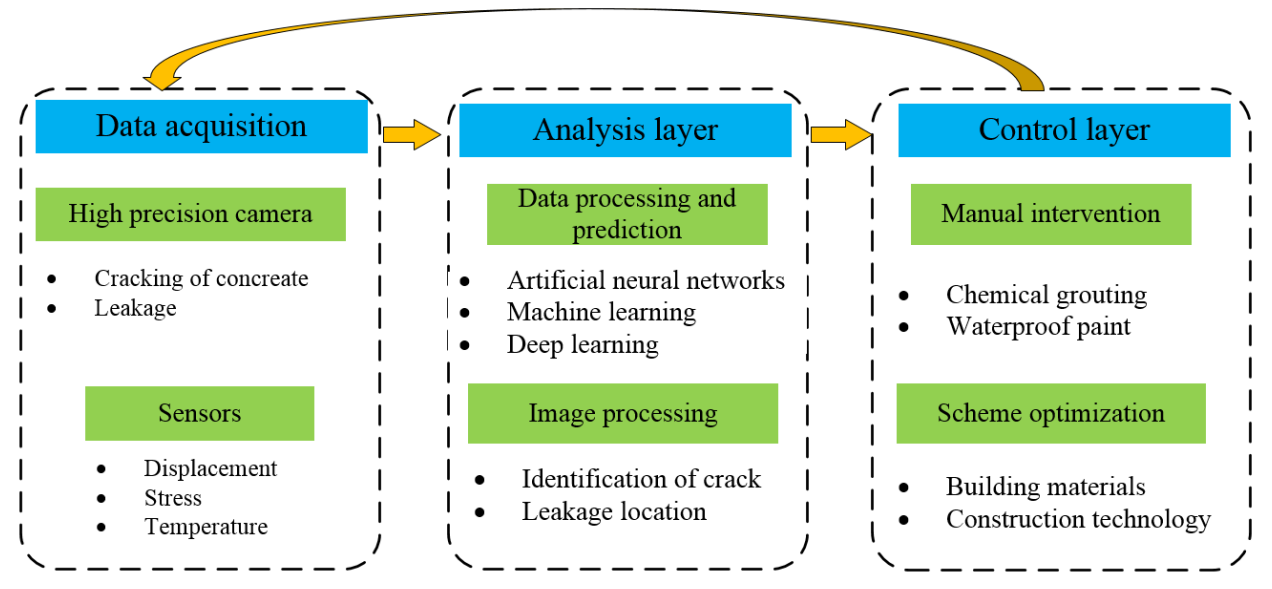

Figure 5. An application framework for smart technology in under-lake tunnels

\section{Conclusions}

China is at a critical stage in the process of urbanization, and the problem of urban traffic congestion is gradually emerging and becoming increasingly serious. The construction of the lake tunnel not only improves the urban road network system but also becomes a unique landscape of the city.

Considering the special geological environment of the lake tunnel, the lake tunnel often has problems such as water seepage and concrete cracking. Smart technology has been successfully applied in data processing and tunnel deformation prediction. This article proposes data acquisition through high-definition cameras, sensors, and other detection devices, and then uses intelligent algorithms for data analysis and processing. On the basis of that, the leakage and concrete cracking in the whole life cycle of tunnel construction and operation can be controlled.

\section{Acknowledgements}

This research is supported by the Fundamental Research Funds for the Central Universities of China (No. PA2019GDPK0089), the Open Fund of the Key Laboratory of Rock Mechanics in Hydraulic Structural Engineering, Ministry of Education, Wuhan University 
(No. RMHSE1604), and Anhui Natural Science Youth Fund (No. 1908085QE216). These supports are gratefully acknowledged.

\section{References}

1. Davies O, Rouainia M, Glendinning S, et al. Investigation of a pore pressure driven slope failure using a coupled hydro-mechanical model[J]. Engineering geology, 2014, 178: 70-81.

2. Liu G, Sun WC, Lowinger SM, Zhang ZH, Huang M, Peng J. Coupled flow network and discrete element modeling of injection-induced crack propagation and coalescence in brittle rock. Acta Geotechnica. 2019;14(3):843-868.

3. Darabi A, Ahangari K, Noorzad A, et al. Subsidence estimation utilizing various approaches-A case study: Tehran No. 3 subway line[J]. Tunnelling and Underground Space Technology, 2012, 31: 117-127.

4. Li S, He P, Li L, et al. Gaussian process model of water inflow prediction in tunnel construction and its engineering applications[J]. Tunnelling and Underground Space Technology, 2017, 69: 155-161.

5. Mahdevari S, Haghighat H S, Torabi S R. A dynamically approach based on SVM algorithm for prediction of tunnel convergence during excavation[J]. Tunnelling and Underground Space Technology, 2013, 38: 59-68.

6. Goh, A.T.C., Hefney, A.M., 2010. Reliability assessment of EPB tunnel-related settlement. Geomech. Eng. 2 (1), $57-69$.

7. China Building Waterproof Association, Beijing Zero Market Research and Analysis Company. National Building Leakage Survey Project Report in 2013, 2013. 